TOPOLOGICAL ALGEBRAS, THEIR APPLICATIONS,

AND RELATED TOPICS

BANACH CENTER PUBLICATIONS, VOLUME 67

INSTITUTE OF MATHEMATICS

POLISH ACADEMY OF SCIENCES

WARSZAWA 2005

\title{
POLYNOMIALS IN THE VOLTERRA AND RITT OPERATORS
}

\author{
DASHDONDOG TSEDENBAYAR \\ UFR Mathématiques, Université de Lille I \\ 59655 Villeneuve d'Ascq Cedex, France \\ E-mail: Dashdondog.Tsedenbayar@agat.univ-lille1.fr \\ and \\ Darkhan Uul Aimag \\ Shuudangiin Salbar 1, P.O. Box 100, Mongolia \\ E-mail:tsdnbr@yahoo.com \\ JAROSLAV ZEMÁNEK \\ Institute of Mathematics, Polish Academy of Sciences \\ Śniadeckich 8, P.O. Box 21, 00-956 Warszawa, Poland \\ E-mail: zemanek@impan.gov.pl
}

\begin{abstract}
We continue the paper [Ts] on the boundedness of polynomials in the Volterra operator. This provides new ways of constructing power-bounded operators. It seems interesting to point out that a similar procedure applies to the operators satisfying the Ritt resolvent condition: compare Theorem 5 and Theorem 9 below.
\end{abstract}

1. Preliminaries. An operator $A$ is called power-bounded if

$$
\sup _{n \geq 0}\left\|A^{n}\right\|<\infty .
$$

Denote by $V$ the classical Volterra operator

$$
(V f)(x)=\int_{0}^{x} f(s) d s, \quad 0<x<1 \text {, on } L^{p}(0,1), 1 \leq p \leq \infty .
$$

The more general Riemann-Liouville integral operator of fractional order $\alpha>0$ is defined by

$$
\left(J^{\alpha} f\right)(x)=\frac{1}{\Gamma(\alpha)} \int_{0}^{x}(x-s)^{\alpha-1} f(s) d s, \quad 0<x<1, \quad \text { on } L^{p}(0,1), 1 \leq p \leq \infty,
$$

where $\Gamma$ is the Euler gamma function. In particular, $V=J^{1}$.

2000 Mathematics Subject Classification: 47A10, 47A35, 47G10.

The paper is in final form and no version of it will be published elsewhere. 
Recall that the Ritt condition for the resolvent $R(\lambda, A)=(\lambda I-A)^{-1}$ of a bounded operator $A$ on a Banach space is

$$
\|R(\lambda, A)\| \leq \frac{\text { const }}{|\lambda-1|}, \quad|\lambda|>1,
$$

which is equivalent to a geometric condition much stronger than the power boundedness of $A$, namely,

$$
\sup _{n \geq 0} n\left\|A^{n}-A^{n+1}\right\|<\infty
$$

has to be added to the power boundedness of $A$, see [NaZe], [Ne]. Examples are the operators $I-J^{\alpha}$ with $0<\alpha<1$, see [Ly]. In particular, the geometric characterization in terms of the behaviour of the powers gives easily the following:

Proposition 1. Let $A$ and $B$ be two commuting Ritt operators. Then their product $A B$ is also a Ritt operator.

If the operator $A$ is merely power-bounded, then the weaker Kreiss condition

$$
\|R(\lambda, A)\| \leq \frac{\text { const }}{|\lambda|-1}, \quad|\lambda|>1
$$

holds, but not conversely in general.

The behaviour of the consecutive powers has been studied in [Ly], [Ne] and [ToZe]. We shall need the following simple facts (see $[\mathrm{Ts}]$ ):

Proposition 2. Let $A$ and $B$ be two commuting power-bounded operators on a Banach space, $0 \leq t \leq 1$. Then the convex combination $t A+(1-t) B$ is a power-bounded operator.

Proposition 3. Let $\sigma(Q)=\{0\}$. If $I-Q$ satisfies the Ritt condition, then so does $I-t Q$ for $t \geq 0$. Consequently, $(1-t) I+t(I-Q)^{2}$ is a Ritt operator for $t \geq 0$.

\section{The results}

LEMMA 4. The resolvent for $a V+b V^{2}$ ( $a$ and $b$ constants) is

$$
\begin{aligned}
\left(R\left(\lambda, a V+b V^{2}\right) f\right)(x)= & \frac{f(x)}{\lambda} \\
& +\frac{1}{\sqrt{a^{2}+4 b \lambda}}\left(\frac{a+\sqrt{a^{2}+4 b \lambda}}{2 \lambda}\right)^{2} \int_{0}^{x} e^{\frac{a+\sqrt{a^{2}+4 b \lambda}}{2 \lambda}}(x-s) f(s) d s \\
& -\frac{1}{\sqrt{a^{2}+4 b \lambda}}\left(\frac{a-\sqrt{a^{2}+4 b \lambda}}{2 \lambda}\right)^{2} \int_{0}^{x} e^{\frac{a-\sqrt{a^{2}+4 b \lambda}}{2 \lambda}}(x-s) f(s) d s
\end{aligned}
$$

where $\lambda \in \mathbb{C} \backslash\{0\}$ and $\sigma\left(a V+b V^{2}\right)=\{0\}$.

Proof. Let $C^{\infty}(0,1)$ be the space of infinitely differentiable functions on $(0,1)$. If $f \in$ $C^{\infty}(0,1)$, then the equation

$$
\left(\left(\lambda I-a V-b V^{2}\right) g\right)(t)=f(t)
$$

is equivalent to the differential equation

$$
\lambda g^{\prime \prime}(t)-a g^{\prime}(t)-b g(t)=f^{\prime \prime}(t)
$$


which is satisfied by

$$
\begin{aligned}
g(x)= & \left(R\left(\lambda, I-a V-b V^{2}\right) f\right)(x) \\
= & \frac{f(x)}{\lambda}+\frac{1}{\sqrt{a^{2}+4 b \lambda}}\left(\frac{a+\sqrt{a^{2}+4 b \lambda}}{2 \lambda}\right)^{2} \int_{0}^{x} e^{\frac{a+\sqrt{a^{2}+4 b \lambda}}{2 \lambda}}(x-s) f(s) d s \\
& -\frac{1}{\sqrt{a^{2}+4 b \lambda}}\left(\frac{a-\sqrt{a^{2}+4 b \lambda}}{2 \lambda}\right)^{2} \int_{0}^{x} e^{\frac{a-\sqrt{a^{2}+4 b \lambda}}{2 \lambda}}(x-s) f(s) d s .
\end{aligned}
$$

Note that $C^{\infty}(0,1)$ is dense in $L^{p}(0,1)(1 \leq p \leq \infty)$.

ThEOREM 5. The operator $I-a V+b V^{2}$ is power-bounded on $L^{2}(0,1)$ for $a>0$ and $b \geq 0$ (and also for $a=b=0$ ).

Proof. Case $0 \leq b \leq a^{2} / 4$. We can write

$$
I-a V+b V^{2}=\left(I-\frac{a-\sqrt{a^{2}-4 b}}{2} V\right)\left(I-\frac{a+\sqrt{a^{2}-4 b}}{2} V\right),
$$

and use [Ts, Theorem 1].

Case $b>a^{2} / 4$. Note that $\left(I-\frac{a t}{2} V\right)^{2}$ is power-bounded for each $t>0$, by [Ts, Theorem 1]. It then follows from Proposition 1 that

$$
(1-\lambda) I+\lambda\left(I-a t V+\frac{a^{2} t^{2}}{4} V^{2}\right)=I-\lambda a t V+\frac{\lambda a^{2} t^{2}}{4} V^{2}
$$

is power-bounded for $0<\lambda<1$. So, $t=1 / \lambda$ with $t=4 b / a^{2}>1$ proves the claim.

Proposition 6. The operator $I-a V+z V^{2}(z \in \mathbb{C})$ is not power-bounded on $L^{2}(0,1)$, for $a<0$, and also for $a>0$ and $z \in \mathbb{C} \backslash[0, \infty)$, or $a=0$ and $z \neq 0$.

Proof. Using Lemma 4 we obtain

$$
\begin{aligned}
& -\left(R\left(\lambda, I-a V-z V^{2}\right) f\right)(x)=\left(R\left(1-\lambda, a V+z V^{2}\right) f\right)(x) \\
& =\frac{f(x)}{1-\lambda}+\frac{1}{\sqrt{a^{2}+4 z(1-\lambda)}}\left(\frac{a+\sqrt{a^{2}+4 z(1-\lambda)}}{2(1-\lambda)}\right)^{2} \int_{0}^{x} e^{\frac{a+\sqrt{a^{2}+4 z(1-\lambda)}}{2(1-\lambda)}}(x-s) f(s) d s
\end{aligned}
$$

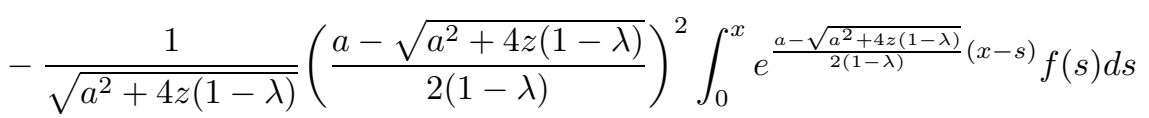

where $\lambda \neq 1$. Analyzing the behaviour of these expressions as $\lambda \rightarrow 1_{+}$, we see that the resolvent $R\left(\lambda, I-a V-z V^{2}\right)$ does not satisfy the Kreiss condition on $L^{2}(0,1)$. See also [Ts, Theorem 3].

THEOREM 7. Let $m \geq 1$ be fixed. The operator

$$
L_{m}(V)=\sum_{k=0}^{m}\left(\begin{array}{c}
m \\
k
\end{array}\right)(-1)^{k} \frac{V^{k}}{k !}
$$

is power-bounded on $L^{2}(0,1)$.

Proof. Recall that the zeros of the Laguerre polynomials $L_{m}(\cdot)$ are real, positive and simple (see [MaOb, p. 84] or [Sz, p. 122]). Suppose that $a_{1}, a_{2}, \ldots, a_{m}$ are the zeros of the Laguerre polynomial $L_{m}$. We can write 


$$
\begin{aligned}
m ! L_{m}(V) & =\left(a_{1}-V\right)\left(a_{2}-V\right) \ldots\left(a_{m}-V\right) \\
& =\left(I-\frac{1}{a_{1}} V\right)\left(I-\frac{1}{a_{2}} V\right) \ldots\left(I-\frac{1}{a_{m}} V\right) \prod_{i=1}^{m} a_{i} .
\end{aligned}
$$

It is clear that $\prod_{i=1}^{m} a_{i}=m$ !. Hence $L_{m}(V)$ is power-bounded by [Ts, Theorem 1$]$.

TheOREM 8. The operator $I-V^{1 / 2}+b V$ is power-bounded on $L^{2}(0,1)$, for $b \in \mathbb{R}$.

Proof. Case $0 \leq b \leq 1 / 4$. We can write

$$
I-V^{1 / 2}+b V=\left(I-\frac{1+\sqrt{1-4 b}}{2} V^{1 / 2}\right)\left(I-\frac{1-\sqrt{1-4 b}}{2} V^{1 / 2}\right),
$$

and use Proposition 3. Note that $V^{1 / 2}=J^{1 / 2}$, hence $I-V^{1 / 2}$ is a Ritt operator.

Case $b>1 / 4$. It follows from Proposition 2, and from the power boundedness of $\left(I-\frac{t}{2} V^{1 / 2}\right)^{2}, t>0$ (see Proposition 3$)$, that

$$
(1-\lambda) I+\lambda\left(I-t V^{1 / 2}+\frac{t^{2}}{4} V\right)=I-\lambda t V^{1 / 2}+\frac{\lambda t^{2}}{4} V
$$

is power-bounded for $0<\lambda<1$. So, $\lambda=1 / t$ with $t=4 b>1$ proves the claim.

Case $b<0$. It follows from Proposition 2, the power boundedness of $I-a V^{1 / 2}(a>0$, see Proposition 3$)$ and $I-t V(t>0,[\mathrm{Ts}$, Theorem 1]) that

$$
(1-\lambda)\left(I-a V^{1 / 2}\right)+\lambda(I-t V)=I-a(1-\lambda) V^{1 / 2}-\lambda t V
$$

is power-bounded for $0<\lambda<1$. We choose $a=1 /(1-\lambda)$, with $0<\lambda=-b / t<1$, which is possible for a sufficiently large $t>0$. The proof is complete.

TheOREM 9. Let $\sigma(Q)=\{0\}$. If $I-Q$ is a Ritt operator, then so is the operator $I-$ $a Q+b Q^{2}$ for $a>0$ and $b \geq 0$ (and also for $a=b=0$ ).

Proof. If $a^{2} \geq 4 b \geq 0$, we can write

$$
I-a Q+b Q^{2}=\left(I-\frac{a-\sqrt{a^{2}-4 b}}{2} Q\right)\left(I-\frac{a+\sqrt{a^{2}-4 b}}{2} Q\right),
$$

where both the factors are Ritt operators, by Proposition 3, hence so is their product, by Proposition 1.

Suppose that $0<a^{2}<4 b$. Let $0<s<1$ and $t>0$. By Proposition 3,

$$
(1-s) I+s\left(1-\frac{a t}{2} Q\right)^{2}=I-a s t Q+\frac{a^{2} s t^{2}}{4} Q^{2}
$$

is a Ritt operator. Choosing $s=1 / t$ with $t=4 b / a^{2}>1$, we get the result.

Proposition $10([\mathrm{Al}])$. Let $\sigma(Q)=\{0\}$. If the operators $I-Q$ and $I+Q$ are powerbounded, then $Q=0$.

Proof. We can write

$$
Q=Q\left(\frac{I-Q+I+Q}{2}\right)^{n}=\frac{1}{2^{n}} \sum_{k=0}^{n}\left(\begin{array}{l}
n \\
k
\end{array}\right)(I-Q)^{n-k} Q(I+Q)^{k} .
$$


Observe that, for large $n$, either $(I-Q)^{n-k} Q$ or $Q(I+Q)^{k}$ is small, by [Es, Theorem 9.1], while the remaining operator powers (actually both $(I+Q)^{k}$ and $\left.(I-Q)^{n-k}\right)$ are bounded, by assumption. It follows that $Q=0$.

\section{Remarks}

REMARK 11. Let

$$
M_{n}(T)=\frac{I+T+\ldots+T^{n-1}}{n} .
$$

The operator $I-V$ is not power-bounded on $L^{1}(0,1)\left(\left\|(I-V)^{n}\right\|\right.$ is of order $\left.n^{1 / 4}\right)$, but $\left\|M_{n}(I-V)\right\|$ is bounded; see $([\mathrm{Hi}]$, [ToZe] $)$. It can be shown that $\left\|M_{n}(I-t V)\right\|$ is bounded, with respect to $n$, for each fixed $t>0$. Indeed, an argument similar to that for Proposition 3 (see [Ts, Proposition 2]) shows that the resolvent of the operator $I-t V$, for a fixed $t>0$, remains uniformly Abel bounded on the half-line $\lambda>1$, which is equivalent to the Cesàro boundedness of $I-t V$ (see [MoSaZe, Theorem 3.1]). Thus, we see one more advantage of the resolvent characterizations of various geometric properties of the powers.

REMARK 12. Observe that the power-boundedness in Theorem 8 for $b<0$ is due to the fact that the operator $I-V^{1 / 2}$ satisfies the Ritt condition (which makes it possible to use Proposition 3).

REMARK 13. In Theorem 5 , for $a>0$ and $b>a^{2} / 4$, the operator is a product of two operators of the form $I-z V$, with $z \notin \mathbb{R}$, that are not power-bounded by [Ts, Theorem 1]. Nevertheless their product is power-bounded.

REMARK 14. Let $\sigma(Q)=\{0\}$. Suppose that the operators $I-Q$ and $I-Q^{2}$ are powerbounded. Does it follow that $I-Q+t Q^{2}$ is power-bounded for $t \in \mathbb{R}$ ? This would be a generalization of Theorem 8 . What about the operators in Theorem 9 , for other values of $a$ and $b$ ?

REMARK 15. Let $m$ be fixed. Observe that the operator $L_{m}\left(J^{\alpha}\right)$, for $0<\alpha<1$, satisfies the Ritt condition on $L^{p}(0,1)$, for $1 \leq p \leq \infty$, by [Ly], Propositions 1 and 3 , and the proof of Theorem 7, but not for $\alpha=1$ and $m=1$. However, by Theorem 7 and [Es, Theorem 9.1] we know that

$$
\lim _{k \rightarrow \infty}\left\|L_{m}(V)^{k}-L_{m}(V)^{k+1}\right\|=0 .
$$

What is the rate of this convergence? Does it depend on $m$ ?

Remark 16. Suppose that $A$ satisfies the Kreiss condition. Does it follow that also $A^{2}$ is a Kreiss operator?

Acknowledgments. The authors are grateful to Professor Mostafa Mbekhta for interesting discussions, and the University of Lille 1 for support and hospitality. 


\section{References}

[Al] G. R. Allan, Sums of idempotents and a lemma of N. J. Kalton, Studia Math. 121 (1996), 185-192.

[Es] J. Esterle, Quasimultipliers, representations of $H^{\infty}$, and the closed ideal problem for commutative Banach algebras, in: Radical Banach Algebras and Automatic Continuity (Long Beach, CA, 1981), J. M. Bachar, W. G. Bade, P. C. Curtis Jr., H. G. Dales, and M. P. Thomas (eds.), Lecture Notes in Math. 975, Springer, 1983, 66-162.

[Hi] E. Hille, Remarks on ergodic theorems, Trans. Amer. Math. Soc. 57 (1945), 246-269.

[Ly] Yu. Lyubich, The single point spectrum operators satisfying Ritt's resolvent conditions, Studia Math. 145 (2000), 135-142.

[MaOb] W. Magnus and F. Oberhettinger, Formulas and Theorems for the Functions of Mathematical Physics, Chelsea, New York, 1954.

[MoSaZe] A. Montes-Rodríguez, J. Sánchez-Álvarez and J. Zemánek, Uniform Abel-Kreiss boundedness and the extremal behaviour of the Volterra operator, Proc. London Math. Soc., to appear.

[NaZe] B. Nagy and J. Zemánek, A resolvent condition implying power boundedness, Studia Math. 134 (1999), 143-151.

[Ne] O. Nevanlinna, Resolvent conditions and powers of operators, Studia Math. 145 (2000), 113-134.

[Sz] G. Szegő, Orthogonal Polynomials, Amer. Math. Soc., New York, 1959.

[ToZe] Yu. Tomilov and J. Zemánek, A new way of constructing examples in operator ergodic theory, Math. Proc. Cambridge Philos. Soc. 137 (2004), 209-225.

[Ts] D. Tsedenbayar, On the power boundedness of certain Volterra operator pencils, Studia Math. 156 (2003), 59-66. 